\title{
Annulohypoxylon sp. FPYF3050 produces volatile organic compounds against the pine wood nematode, Bursaphelenchus xylophilus
}

\author{
Hongchang $\mathrm{LI}^{1,2}$, Guiming Dou ${ }^{1}$, Mengge $\mathrm{GAO}^{3}$, Fei ReN ${ }^{4}$, Ruhua $\mathrm{LI}^{1}$, Xingyao ZhanG ${ }^{3}$ and \\ Dong-Hui YAN ${ }^{1,2, *}$ \\ ${ }^{1}$ Research Institute of Forest Ecology, Environment and Protection, Key Laboratory of Forest Protection of State Forestry \\ Administration, Chinese Academy of Forestry, Beijing 100091, China \\ ${ }^{2}$ Co-Innovation Center for Sustainable Forestry in Southern China, Nanjing Forestry University, Nanjing 210037, \\ P.R. China \\ ${ }^{3}$ Research Institute of Forestry New Technology, Chinese Academy of Forestry, Beijing 100091, P.R. China \\ ${ }^{4}$ Experimental Center of Forestry in North China, Chinese Academy of Forestry, Beijing 102300, P.R. China
}

Received: 19 May 2019; revised: 24 July 2019 Accepted for publication: 25 July 2019; available online: 9 October 2019

\begin{abstract}
Summary - Natural volatiles released by the fungus, Annulohypoxylon sp. FPYF3050, were evaluated against the pine wood nematode (PWN), Bursaphelenchus xylophilus. Our results showed that volatile organic compounds (VOCs) caused 64.1 and $58.4 \%$ mortality of second-stage juveniles (J2) and mixed-stages (eggs, J2, third- and fourth-stage juveniles, and adults) of populations of PWN, but no inhibitive effects were detected on nematode eggs in the experiment. Analysis of the gases within the Petri plate by gas chromatographymass spectrometry (GC-MS) showed a yield of an unique volatile with dominant 1,8-cineole in $77.4 \%$ relative area (RA) after $72 \mathrm{~h}$ treatment of nematodes with Annulohypoxylon sp. FPYF3050 and Botrytis cinerea. The commercial 1,8-cineole at concentrations of 2, 5, 10 and $15 \mu \mathrm{l} \mathrm{ml}^{-1}$ was applied to examine nematicidal activity. The results showed that 1,8-cineole had a 40-100\% inhibition on the nematode eggs during $48 \mathrm{~h}$ treatment, more than $82.9 \%$ mortality of $\mathrm{J} 2$ after $24 \mathrm{~h}, 48 \mathrm{~h}$ and $96 \mathrm{~h}$, and $18.7-91.9 \%$ mortality of the mixed-stage population, depending on the period after exposure. This result indicates that 1,8-cineole in the volatile gas emissions of Annulohypoxylon sp. FPYF3050 may play a crucial inhibitory effect on the pine wood nematode The nematicidal volatile gas from fungi may provide a useful biocontrol agent for controlling B. xylophilus.
\end{abstract}

Keywords - 1,8-cineole, biological control, fungus, nematicidal activity, pine wilt disease.

Pine wilt disease caused by the pine wood nematode (PWN), Bursaphelenchus xylophilus, was first reported by Cheng in 1982 in China (Cheng et al., 1983). At that time, the disease was limited to the site of Sun Yat-sen's Mausoleum in Nanjing, where the infestation covered 200 $\mathrm{hm}^{2}$ and affected 256 trees of Pinus thunbergii. However, it has extended to 18 provinces during the last 30 years, damaged much of China's forest resources and caused great economic losses. Currently, more effective means are expected to be found to control B. xylophilus.

Fungi are unique candidates for using in an 'inundation' biocontrol strategy (Jackson et al., 2009). Some types of fungi have been widely used as agents in the biocontrol of plant-parasitic nematodes. Nematode-trapping

* Corresponding author, e-mail: yandh@caf.ac.cn fungi have evolved complex trapping devices to capture and consume nematodes (Lopez-Llorca et al., 2007; Ulzurrun \& Hsueh, 2018). Nematode-endoparasite fungi produce numerous lunate conidia that adhere to nematodes, killing them (Shih \& Tzean, 1999). Other fungi produce toxic secondary metabolites that immobilise and kill nematodes (Freire et al., 2012; Yang et al., 2012; Pimenta et al., 2017).

Volatile organic compounds (VOCs) can permeate the air and travel a long distance, which makes fungal VOCs a potential, useful and additional tool in biocontrol strategies (Morath et al., 2012). Some studies suggested that the VOCs of some endophytic fungi were able to inhibit the growth of plant pathogens by augmenting host 
defence responses against pathogens (Macías-Rubalcava et al., 2010). A series of endophytic fungi were recently found to produce VOCs that inhibited nematodes (Freire et al., 2012; Yang et al., 2012; Pimenta et al., 2017). Pimenta et al. (2017) reported that VOCs of 28 endophytic fungal isolates immobilised more than $76 \%$ of secondstage juveniles (J2) of Meloidogyne incognita. The fungal species Epicoccum nigrum and Schizophyllum commune produced VOCs that immobilised J2 after $6 \mathrm{~h}$ exposure. VOCs emitted by 13 fungi isolated from coffee roots caused more than $40 \%$ immobility of $\mathrm{J} 2 \mathrm{M}$. incognita (Pimenta et al., 2017). However, there is no information on the action of volatile gases from endophytic fungi on pine wood nematodes.

Volatile terpenoids are a class of VOCs produced by many fungal species (Shaw et al., 2015), and they are usually monoterpene and sesquiterpene. Many monoterpenoids showed suppression of nematodes and were more toxic to some nematodes than the commercial nematicide Oxamyl (Tsao \& Yu, 2000). It has been reported that 1,8-cineole, a monoterpene, had 63.5, 47.0 and $46.0 \%$ nematicidal action on M. incognita, Pratylenchus vulnus and Xiphinema index, respectively (Avato et al., 2017).

In this study of the endophytic fungal volatiles on important plant pathogens, we found that the volatiles of $A n$ nulohypoxylon sp. FPYF3050 produced a unique volatile with abundance of 1,8-cineole. We investigated the effects of the VOCs emitted by the fungus, Annulohypoxylon sp. FPYF3050, on different life stages of PWN, including egg, J2, and mixed-stage that contained eggs, J2, third- (J3) and fourth-stage (J4) juveniles and adults. We also evaluated the nematicidal activity of different concentrations of commercial 1,8-cineole on PWN. Our results demonstrated that VOCs of Annulohypoxylon sp. FPYF3050 had an obvious effect on PWN and could be a potentially useful biocontrol agent against the PWN.

\section{Materials and methods}

\section{FUNGAL AND NEMATODE MATERIAL}

Annulohypoxylon sp. FPYF3050 was isolated from branches of the shrub plant Neolitsea pulchella (Wang et al., 2017). The strain (Nxy61) of PWN in the present study was isolated from P. massoniana trees in Ningbo, P.R. China (Liang et al., 2013), in October 1992, and maintained on Botrytis cinerea on corn-meal agar (CMA) in the culture collection of the Chinese Academy of Forestry (Beijing, P.R. China). Potato dextrose agar (PDA; potato $200 \mathrm{~g}$, glucose $20 \mathrm{~g}$, agar $25 \mathrm{~g}$, and $\mathrm{H}_{2} \mathrm{O} 1000$ $\mathrm{ml}$ ) medium in a Petri dish (diam. $90 \mathrm{~mm}$ ) was used for incubating fungi, Annulohypoxylon sp. FPYF3050 and $B$. cinerea in this experiment.

\section{COLLECTING B. XYLOPHILUS EGGS AND J2}

PWN (Nxy61) were cultured on B. cinerea in the dark at $25^{\circ} \mathrm{C}$. After 5 days, $20 \mathrm{ml}$ of dd $\mathrm{H}_{2} \mathrm{O}$ was added to wash off the nematodes from the PDA plate to obtain mixed stages (eggs, J2, J3, J4 and adults). The nematode suspension was partly used in the test on mixedstage nematodes and partly for collecting the eggs. To collect eggs, the nematode suspension was transferred to a new and clean glass Petri dish ( $90 \mathrm{~mm}$ diam.) and left for 20 mins at $25^{\circ} \mathrm{C}$ for eggs to settle on the base of the Petri dish, where they adhered to the glass due to glycoproteins in their surfaces (Shinya et al., 2009). After carefully removing the water and worms, the eggs were rinsed repeatedly with sterile water and transferred to a centrifuge tube to obtain the pure eggs. To obtain synchronous development of $\mathrm{J} 2$, the collected eggs were kept in sterile water in an incubator at $25^{\circ} \mathrm{C}$ for $24 \mathrm{~h}$ (Shinya et al., 2009; Zhu et al., 2016).

\section{NEMATICIDAL ACTIVITY TESTS FOR FUNGAL VOCS}

The nematocidal activity of VOCs was determined by the methods previously described (Strobel et al., 2001; Yan et al., 2018). A $2 \mathrm{~cm}$ wide central strip was removed from the PDA medium in a Petri dish $(90 \mathrm{~mm}$ diam.). Then, the PDA medium was divided into two semicircular PDA medium sheets (Fig. 1A).

For the test on eggs, a $2 \mathrm{ml}$ Eppendorf centrifuge tube cover was inverted onto one half-moon of the agar, and Annulohypoxylon sp. FPYF3050 was inoculated onto the other half-moon of the agar. The Petri dish was incubated in a growth chamber in the dark at $25^{\circ} \mathrm{C}$ for 5 days. An egg suspension $(100 \mu \mathrm{l})$, which contained 100 eggs, was introduced into the tube cover and left in the dark at $25^{\circ} \mathrm{C}$ for $\mathrm{J} 2$ to hatch. The eggs were checked at 6 , $12,18,24,30$ and $48 \mathrm{~h}$ intervals. The same treatment without Annulohypoxylon sp. FPYF3050 served as the control (Fig. 1B).

For the synchronously developed $\mathrm{J} 2$ and mixed-stage test, before the bioassay experiment, Annulohypoxylon sp. FPYF3050 and B. cinerea were inoculated onto one halfmoon of an agar individually. After 5 days, two halves, one with $B$. cinerea and the other with Annulohypoxylon sp. FPYF3050, were recombined into a new agar in a Petri dish on the fifth day and 2000 synchronously developed J2 or mixed-stage nematodes in $0.2 \mathrm{ml}$ suspension were then 


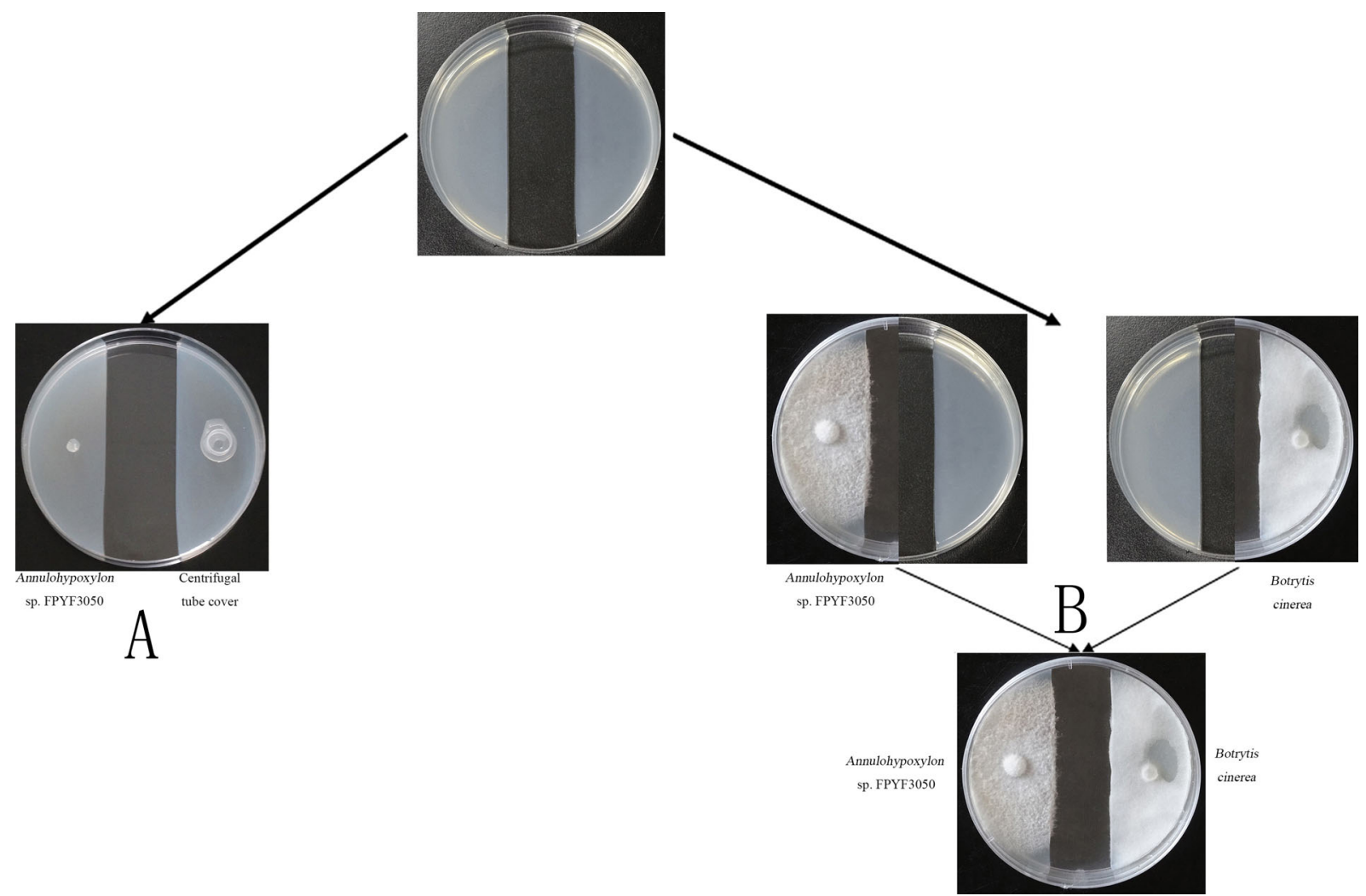

Fig. 1. Assay methods for nematicidal volatiles emitted by fungi. A: Experimentation on eggs. Annulohypoxylon sp. FPYF3050 was inoculated on the left and the centrifugal tube cover was on the right. Five days later, an egg suspension was injected into the tube cover; B: Experimentation using second-stage juveniles (J2) of Bursaphelenchus xylophilus, and mixed-stage nematodes. Annulohypoxylon sp. FPYF3050 and Botrytis cinerea were cultured for 5 days and combined in the same Petri dish, Annulohypoxylon sp. FPYF3050 was positioned on the left and the $B$. cinerea fungus on the right; 2000 nematodes were inoculated in the centre of $B$. cinerea, which caused a pit to form in the centre.

inoculated on the colony of $B$. cinerea. A Petri dish with only $B$. cinerea on the half plate served as control. The method was used for surveying the effect of volatiles on PWN during the whole study (Fig. 1B).

In the previous study, the most abundant 1,8-cineole of Annulohypoxylon sp. FPYF3050 occurred in the 3 days between days 5 and 7 after the endophytic fungus inoculation in the PDA of the Petri dish in the dark at $25^{\circ} \mathrm{C}$ (Wang et al., 2017). Previous work showed reduction in the survival of $\mathbf{J} 2$ after $72 \mathrm{~h}$ of exposure to VOCs emitted by other fungi (Yang et al., 2012; Pimenta et al., 2017), so we chose $72 \mathrm{~h}$ as the treatment time. The colony of $B$. cinerea was established by inoculating from a $0.5 \mathrm{~cm}$ plug of $B$. cinerea and maintained for 5 days; our pilot study found that $2000 \mathrm{PWN}$ of mixed-stage or J2 took $72 \mathrm{~h}$ to exhaust such a colony. Hence, $2000 \mathrm{~J} 2$ were transferred into the $B$. cinerea colony of each treatment group to ensure that $B$. cinerea was digested completely in 3 days. The same treatment was also used for the mixed-stage experiment. After the $72 \mathrm{~h}$ treated with VOCs of Annulohypoxylon sp. FPYF3050, the nematodes were washed off from the PDA with $20 \mathrm{ml}$ of sterile water. The total volume of $20 \mathrm{ml}$ nematode suspension was collected in a $50 \mathrm{ml}$ centrifuge tube. A $100 \mu \mathrm{l}$ suspension was removed from the $50 \mathrm{ml}$ centrifuge tube for counting the nematodes under a microscope (Nikon Eclipse E100), with ten separate counts at a magnification of $4 \times$. The nematodes from the control were processed in the same way. The toxicity level of VOCs was defined by the mean mortality of the nematodes.

$$
\text { Mortality }=(1-a / b) \times 100 \%
$$

where $a$ is the number of nematodes and eggs in a treatment plate; and $b$ is the number of nematodes and eggs in the control plate. 


\section{ANALYSIS OF MIXED VOLATILE COMPOUNDS IN CO-CULTURING TWO FUNGI IN PDA AFTER $72 \mathrm{H}$}

For analysing fungal VOCs in the air space above plates, the gases were collected by SPME-GC-MS. This investigation was done on four groups: i) Annulohypoxylon sp. FPYF3050 and B. cinerea with nematodes; $i i) A n$ nulohypoxylon sp. FPYF3050 and B. cinerea; iii) Annulohypoxylon sp. FPYF3050; and iv) B. cinerea. The fungus of each group grew a colony for 5 days with the same culture conditions as described above. After treatment for 3 days, the compounds released in the headspace of the plates were checked using the GC-MS protocol as described in detail by Wang et al. (2017). In brief, a solid phase microextraction (SPME) syringe (DVBPDMS-Carboxen 50/30, Supelco) was inserted into the headspace inside the Petri plate, for $40 \mathrm{~min}$, through a small hole $(0.5 \mathrm{~mm}$ diam.) drilled on the side of the Petri plate. After exposure to the VOCs, the syringe was inserted into the injection port of the TRACE DSQ inlet, a Thermo Finnigan gas chromatograph and an HP-5 column (5\% phenyl-95\% dimethylsiloxane) with dimensions $30 \mathrm{~m} \times 0.25 \mathrm{~mm} \times 0.25 \mu \mathrm{m}$. The MS was scanned at a rate of 5 scans s $^{-1}$ over a mass range of 41-560 amu. The desorption time was $1 \mathrm{~min}$. The temperature program of the GC oven was: $40^{\circ} \mathrm{C}$ for 2 min and then the temperature increased to $220^{\circ} \mathrm{C}$ at a rate of $5^{\circ} \mathrm{C} \mathrm{min}^{-1}$. PDA Petri plates not inoculated with the strain were used as the control to identify and deduct those compounds contributed by the medium. The tentative identification was made with GC against the NIST compounds library. For comparing mass spectra, we considered only peaks with a spectra similarity greater than $60 \%$.

\section{EFFECT OF COMMERCIAL 1,8-CINEOLE ON $B$. XYLOPHILUS}

1,8-cineole was emitted by Annulohypoxylon sp. FPYF3050 and accounted for $77 \%$ RA in mixing volatiles. Hence, 1,8-cineole could be posited to play a critical role in the reduction of PWN. In order to prove this, appropriate amounts of commercial 1,8-cineole (Macklin) were added to a $0.5 \%$ water solution of Triton X-100 (Acros Organics) to prepare 4, 10, 20 and $30 \mu 1 \mathrm{ml}^{-1} 1$,8-cineole solutions for PWN inhibition tests. Batches of 100 eggs suspended in $0.05 \mathrm{ml}$ distilled water was placed in $0.2 \mathrm{ml}$ Eppendorf vials. A $0.05 \mathrm{ml}$ volume of each concentration of 1,8-cineole solution was poured into each vial containing the nematode suspension to obtain final concentrations of 2, 5, 10 and $15 \mu 1 \mathrm{ml}^{-1}$ and mixed immediately by pipetting. After 24,48 or $96 \mathrm{~h}$ of treatment with each con- centration of the test solutions, the survival nematodes in the Eppendorf vials were checked under the microscope. A $0.5 \%$ Triton X-100 solution was included as control. This method also was used to evaluate the nematicidal activity on $\mathrm{J} 2$ and mixed-stages with 100 individuals in the test solution. All of the treatments and controls were cultured in the dark at $25^{\circ} \mathrm{C}$.

$$
\text { Inhibition of hatching }=(1-(a-c) / b) \times 100 \% \text {. }
$$

Where $a$ is the number of eggs containing unhatched $\mathrm{J} 2$ after the treatment; $b$ is the number of $\mathbf{J} 2$ in the water control; and $c$ is the number of eggs containing unhatched $\mathrm{J} 2$ in the Triton X-100 control.

$$
\text { Mortality of } \mathrm{J} 2=(1-a / b) \times 100 \% .
$$

Where $a$ is the number of nematodes after the treatment; and $b$ is the number of nematodes in the water control.

In the experiment on mixed-stage, we compared the initial number of nematodes in each treatment with the nematodes after treatment to calculate the mortality.

$$
\text { Mortality of mixed-stage }=(1-a / b) \times 100 \% \text {. }
$$

Where $a$ is the number of nematodes after treatment; and $b$ is the number of nematodes before treatment.

\section{STATISTICAL ANALYSIS}

To achieve greater accuracy, all of the nematodes used in treatment and control came from the same centrifuge tube. The population size of nematodes was counted under a microscope (Nikon Eclipse E100) at a magnification of $4 \times$ by the same tester. Three replicates were conducted for each treatment and control, and each in vitro and Petri dish experiment was repeated three times. SPSS 25 was used to carry out an analysis of variance (ANOVA) of the data. Independent sample $t$-test was applied for evaluation of results; $P<0.05$ was used as the level of significance. Values shown are mean $\pm \mathrm{SD}$.

\section{Results}

\section{TOXICITY OF VOCS EMITTED BY ANNULOHYPOXYLON SP. FPYF3050 TO B. XYLOPHILUS}

In the assay of the volatile gas substances produced by Annulohypoxylon sp. FPYF3050 against the PWN eggs, the number of eggs with unhatched $\mathrm{J} 2$ remaining in the treated group and control group were significantly different only at $18 \mathrm{~h}(P=0.008)$ and $30 \mathrm{~h}(P=0.028)$, indicating the inhibitory effect of volatile gases on PWN 


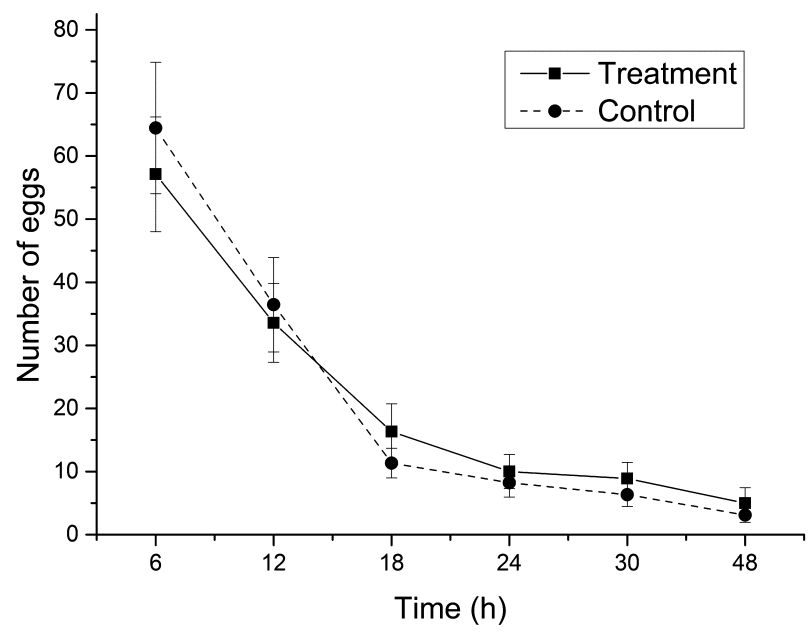

Fig. 2. Number of unhatched $\mathrm{J} 2$ (number of eggs) of Bursaphelenchus xylophilus after exposure for 6-48 h to volatile organic compounds (VOCs) from Annulohypoxylon sp. FPYF3050 and control with no VOCs. Error bars $=\mathrm{SD}$ of the mean.

eggs in this experiment was limited. At $14 \mathrm{~h}$, the number of eggs with unhatched $\mathrm{J} 2$ in the treatment group was slightly greater than that in the control group, indicating minor inhibition (Fig. 2).

In the experiment on synchronously developed $\mathrm{J} 2$, more nematodes survived in the control (2526) than in the $\mathrm{J} 2$ treatment (913). This $64.1 \%$ reduction indicated a strong nematicidal effect of the VOCs released by Annulohypoxylon sp. FPYF3050 on J2 of PWN after 3 days of treatment $(P<0.05)$ (Fig. 3A).

For the effect of the fungal VOCs on the mixed-stage nematode population, 2211 nematodes survived in the control, while far fewer (945) nematodes survived in the treatment, a reduction of $58.4 \%$ after $72 \mathrm{~h}$ treatment (Fig. 3A). The number of $\mathrm{J} 2$ decreased the most, with a decrease of $69.5 \%$, which was basically consistent with the result of the experiment on synchronously developed $\mathrm{J} 2$ above. The mortality of $\mathrm{J} 3$ and adults was 49.5 and $5.03 \%$, respectively. The impact on $\mathrm{J} 4$ was the weakest with only $26.5 \%$ mortality (Fig. 3B).

\section{ANALYSIS OF THE COMPONENTS OF VOCS IN FOUR TREATMENTS}

We analysed the components of VOCs in four treatments after a 3-day period: i) Annulohypoxylon sp. FPYF3050 and B. cinerea with nematodes; ii) Annulohypoxylon sp. FPYF3050 and B. cinerea; iii) Annulohypoxylon sp. FPYF3050; and $i v)$ B. cinerea. The major compound products in treatment $i$ ) were identified as 1,8-cineole, $(+)$-sativene and isocaryophillene, account- ing for $77.4,7.9$ and $7.4 \%$ RA, respectively. The three compounds were also found in $i$ ) with 70.6, 2.5 and 15.9 RA. Only 1,8-cineole was detected as a major product in iii) with $66.3 \%$ RA. The GC/MS analysis results of the four treatments revealed that there were no obvious products released by $B$. cinerea, and 1,8-cineole emitted by Annulohypoxylon sp. FPYF3050 was the most abundant compound in the VOCs (Table 1).

\section{EFFECT OF COMMERCIAL 1,8-CINEOLE ON PWN}

In order to verify the nematicidal activity of the main product, 1,8-cineole, in VOCs of FPYF3050, a commercial product of 1,8-cineole was used to study its inhibitive effects on hatching, and mortality of $\mathrm{J} 2$ and mixed-stage.

After $24 \mathrm{~h}$ treatment of eggs, the percentage hatching inhibition in the concentrations of 2, 5, 10 and $15 \mu \mathrm{ml}^{-1}$ of the commercial 1,8-cineole were 39.8, 79.1, 87.2 and $92.9 \%$, respectively. The rates were $42.1,94.5,98.0$ and $100 \%$ at $48 \mathrm{~h}$ and $0.8,61.8,77.0$ and $86.7 \%$ at $96 \mathrm{~h}$ (Fig. 4A).

In the experiment of 1,8-cineole against synchronously developed $\mathrm{J} 2$, there was a mortality of more than $82.9 \%$ for any test time and all concentrations (Fig. 4B).

In the experiment with 1,8-cineole on the mixed-stage, the overall mortality with treatment time was from 18.7 to $91.9 \%$ in $2 \mu \mathrm{ml}^{-1} 1,8$-cineole solution. With $5 \mu 1 \mathrm{ml}^{-1}$ 1,8-cineole, nematode mortality was $40.4,54.0$ and $65.4 \%$ at $24 \mathrm{~h}, 48 \mathrm{~h}$ and $96 \mathrm{~h}$, respectively. After $24 \mathrm{~h}, 48 \mathrm{~h}, 96 \mathrm{~h}$ exposure to $10 \mu \mathrm{l} \mathrm{ml}^{-1} 1,8$-cineole solution mortality was $89.2,75.4$ and $56.1 \%$, respectively. Mortality in the $15 \mu \mathrm{l}$ $\mathrm{ml}^{-1} 1,8$-cineole solution ranged from 47.3 to $73.1 \%$ after 24 to $96 \mathrm{~h}$ exposures.

For further analyses of the effects on each development stage in the mixed nematodes experiments, the solution of the commercial 1,8-cineole had a marked effect on PWN J2, resulting in a mortality of almost $100 \%$ in all treatments except $2 \mu \mathrm{l} \mathrm{ml}^{-1}$ at $24 \mathrm{~h}$. J3 had almost $22.0 \%$ mortality after exposure to $2 \mu 1 \mathrm{ml}^{-1}$ of 1,8 -cineole for $24 \mathrm{~h}$, increasing to $100 \%$ at $96 \mathrm{~h}$. With exposure to $5 \mu \mathrm{l} \mathrm{ml} l^{-1}$ solution, $\mathrm{J} 3$ had $36.4-63.6 \%$ mortality. In $10 \mu 1 \mathrm{ml}^{-1}$ solution, J3 had mortalities between 57.7 and $62.8 \%$, increasing with exposure time. Mortalities caused by $15 \mu 1 \mathrm{ml}^{-1}$ of 1,8 -cineole ranged from 54.7 to $62.8 \%$. In the treatment group and the control group of each time and concentration, about ten J4 survived (Supplementary material, Table S1). The numbers of J4 surviving in most groups after treatment did not differ significantly (Fig. 5). The commercial 1,8-cineole displayed strong 

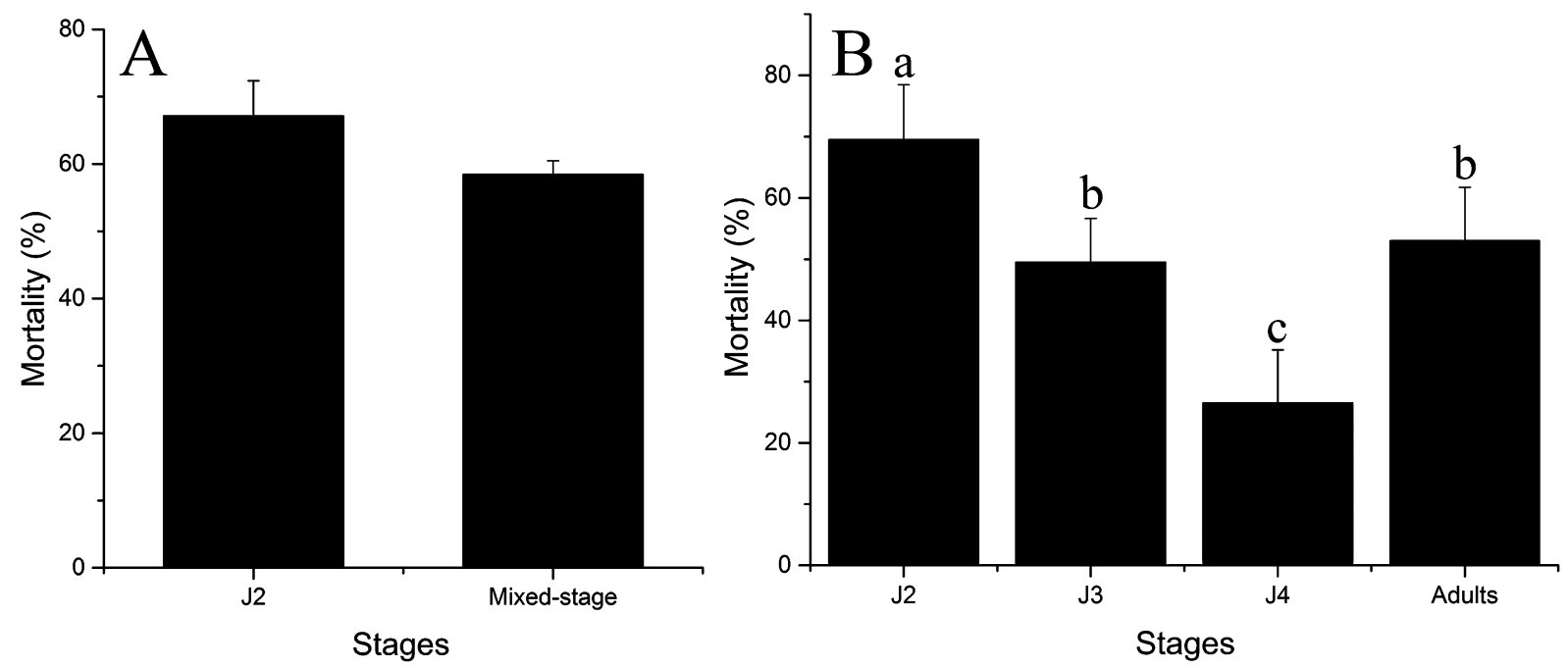

Fig. 3. A: Mortality of Bursaphelenchus xylophilus (mean number of individuals) in the experiments with second-stage juveniles (J2) and mixed-stages after exposure to the volatile organic compounds (VOCs) of Annulohypoxylon sp. FPYF3050. B: Mortality of different stages of Bursaphelenchus xylophilus after exposure to VOCs of Annulohypoxylon sp. FPYF3050. Error bars = SD of the mean. Bars with the same letter are not significantly different based on the independent sample $t$-test. J3 = third-stage juveniles; J4 = fourth-stage juveniles.

Table 1. Volatile organic compounds emitted by fungi as identified by SPME-GC-MS after $72 \mathrm{~h}$ of cultivation. Four Petri plate treatments were prepared: i) Annulohypoxylon sp. FPYF3050 and Botrytis cinerea with nematodes (Bursaphelenchus xylophilus); ii) Annulohypoxylon sp. FPYF3050 and B. cinerea; iii) Annulohypoxylon sp. FPYF3050; and iv) B. cinerea.

\begin{tabular}{|c|c|c|c|c|c|}
\hline & \multirow{2}{*}{$\begin{array}{l}\text { Retention } \\
\text { time }\end{array}$} & \multicolumn{4}{|c|}{ Area $(\%)$} \\
\hline & & $\begin{array}{l}\text { Annulohypoxylon sp. } \\
\text { FPYF3050 and Botrytis } \\
\text { cinerea with nematodes }\end{array}$ & $\begin{array}{c}\text { Annulohypoxylon sp. } \\
\text { FPYF3050 and B. cinerea }\end{array}$ & $\begin{array}{c}\text { Annulohypoxylon sp. } \\
\text { FPYF3050 }\end{array}$ & B. cinerea \\
\hline $\mathrm{CO}_{2}$ & 1.74 & - & - & - & $1.1 \pm 0.7$ \\
\hline Alanine & 1.79 & $0.6 \pm 0.1$ & - & - & - \\
\hline Ethyne, fluoro- & 2.06 & - & - & - & - \\
\hline $\mathrm{N}_{2} \mathrm{O}$ & 2.36 & - & - & - & $0.6 \pm 0.1$ \\
\hline $\begin{array}{l}\text { 3-Heptene, } \\
\text { 2,2,4,6,6-pentamethyl- }\end{array}$ & 9.52 & - & - & - & $8.2 \pm 6$ \\
\hline 1,8-cineole & 10.2 & $77.4 \pm 4.4$ & $70.6 \pm 12.7$ & $66.3 \pm 10.3$ & - \\
\hline$\gamma$-Terpinene & 10.93 & - & $2.3 \pm 0.4$ & $1.6 \pm 0.3$ & - \\
\hline $\begin{array}{l}\text { Sulfurous acid, } \\
\text { cyclohexylmethyl } \\
\text { octadecyl ester }\end{array}$ & 17.37 & - & - & - & $4.8 \pm 2.6$ \\
\hline $\begin{array}{l}\text { 2,4,4,6,6,8,8-Heptamethyl } \\
\text { 2-nonene }\end{array}$ & 1- 17.37 & $1.4 \pm 0.4$ & - & - & $5.5 \pm 3.3$ \\
\hline$(+)$-Sativene & 19.41 & $7.9 \pm 7$ & $2.5 \pm 1.2$ & - & - \\
\hline Isocaryophillene & 20.12 & $7.4 \pm 3$ & $15.8 \pm 5.5$ & - & - \\
\hline
\end{tabular}

The values indicate means $(\%) \pm \mathrm{SD}$ of three replicates. The compounds in the PDA have been removed from the table.

activity against adult nematodes with $28.5,34.4$ and $89.6 \%$ mortality after 24,48 and $96 \mathrm{~h}$ exposure to $2 \mu \mathrm{l}$ $\mathrm{ml}^{-1}$ solutions, respectively; equivalent exposure times of adults to a $5 \mu \mathrm{l} \mathrm{m}^{-1}$ solution resulted in $18.5,46.7$ and $76.5 \%$ mortality, respectively. In addition, the range of mortality after exposure to $10 \mu \mathrm{ml}^{-1}$ solution was 

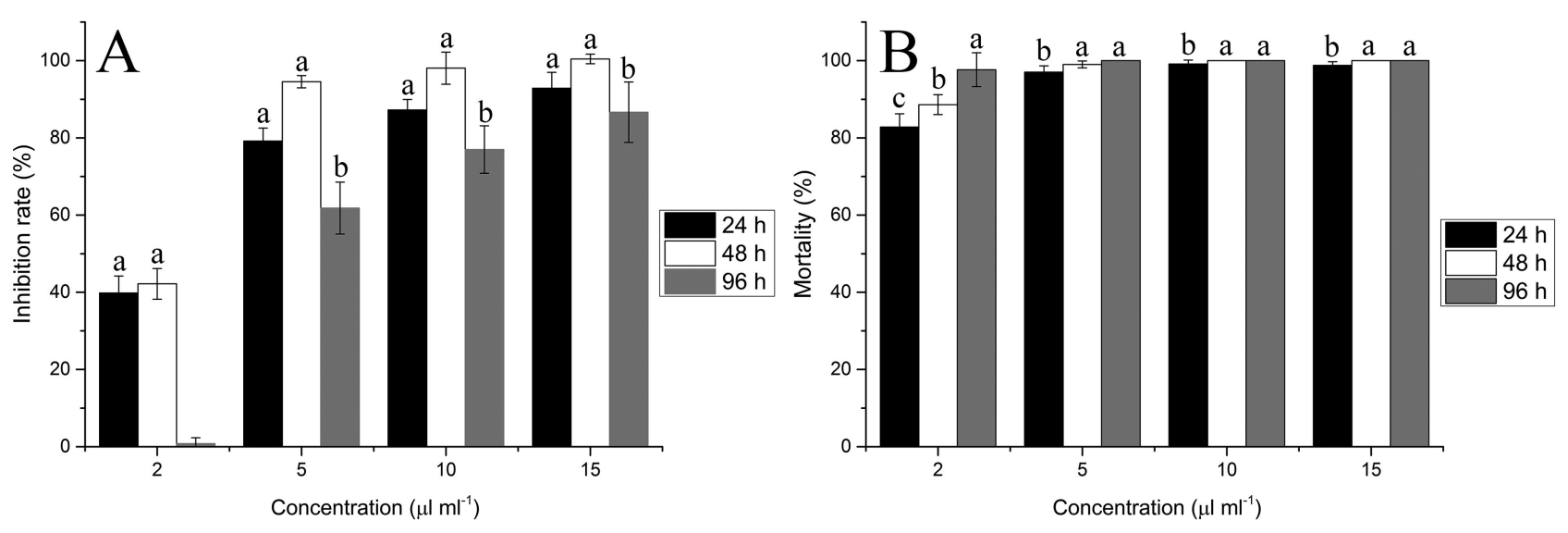

Fig. 4. Over-time hatch inhibition and mortality of Bursaphelenchus xylophilus second-stage juveniles (J2) after immersion in 1,8-cineole solution. A: Hatch inhibition after 24, 48 and $96 \mathrm{~h}$; B: Mortality of J2 after different processing time. Error bars $=$ SD of the mean. Bars with the same letter are not significantly different based on the independent sample $t$-test.

around 38.5-67.0\%. After immersion in the $15 \mu \mathrm{l} \mathrm{ml} \mathrm{l}^{-1}$ solution, mortality percentages were from $53.9-76.5 \%$. The detailed number of nematodes of various ages is given in Table S1 (Supplementary material). In the control group, there was no significant difference in the number of PWN over time and the stages developed normally (Fig. 6).

\section{Discussion}

We found that the VOCs of Annulohypoxylon sp. FPYF3050 had significant nematicidal effects on the juveniles and adults of B. xylophilus. The inhibition of $\mathrm{J} 2$ was particularly prominent. The mortality of synchronously developed $\mathrm{J} 2$ was $64.1 \%$ and the mortality of $\mathrm{J} 2$ in the mixed-stage test was significantly higher than other stages at $69.5 \%$. 1,8-cineole was the dominant component of the volatiles of Annulohypoxylon sp. FPYF3050 with nematicidal activity and its RA accounted for $77.0 \%$. Therefore, we examined the effects of commercial 1,8-cineole on PWN. The results showed that the commercial 1,8-cineole also had a significant inhibitory effect on $\mathrm{J} 2$ under the tested concentration range. With exposure to $2 \mu \mathrm{l} \mathrm{ml}^{-1}$ for $24 \mathrm{~h}$, the mortality of synchronously developed $\mathrm{J} 2$ was greater than that of $\mathrm{J} 2$ in the mixed-stage. The reason may be that the synchronised J2 were in a unified physiological state and were weaker and less resistant to toxic substances, but the $\mathrm{J} 2$ in the mixed-stage batches were in different phases of $\mathbf{J} 2$ development. All the above results indicated that the $\mathrm{J} 2$ was the most sensitive stage to external stimuli, which has been reported for J2 of other plant- parasitic nematodes (Andrés et al., 2012; Pimenta et al., 2017).

Volatile gases caused a $26.5 \%$ mortality of $\mathrm{J} 4$, which was significantly lower than that for other stages, while commercial 1,8-cineole also had a weaker effect on J4 than other stages. The external cortical layer of the cuticle of J4 is thicker than other stages, making J4 more resistant to stress (Giblin-Davis, 1993). Studies had also shown that PWN J4 required more trans-2-hexenal than other stages to achieve a lethal effect (Cheng et al., 2017). Thus, J4 was the most resistant stage to treatment with VOCs. In addition, natural volatile gases from Annulohypoxylon sp. FPYF3050 had a stronger effect than commercial 1,8-cineole on $\mathrm{J} 4$, which suggested that there might be other substances in the volatile gases of $A n$ nulohypoxylon sp. FPYF3050 that affected the PWN. Commercial 1,8-cineole improved the toxicity of other xenobiotics (Rossi \& Palacios, 2015). 1,8-cineole/carvone (0.1-0.4 $\mathrm{mg} \mathrm{ml}^{-1}$ ) caused $100 \% \mathrm{~J} 2$ mortality and $87 \%$ hatch suppression; however, carvone or 1,8-cineole alone showed no nematicidal activity against $M$. javanica (Andrés et al., 2012). In addition, 1,8-cineole is a key element affecting the Ditylenchus destructor that was found in Ajania fruticulosa and A. potaninii essential oils (Liang et al., 2018). Therefore, this nematicidal effect of active volatiles from Annulohypoxylon sp. FPYF3050 may be caused by 1,8 -cineole synergies with other substances.

The inhibitory effect of the commercial 1,8-cineole on the eggs was clear. Avato et al. (2017) also reported that 2, 5, 10,15 $\mu \mathrm{l} \mathrm{ml}^{-1}$ concentrations of 1,8-cineole of had clear hatching inhibitory effects on eggs of $M$. incogni- 

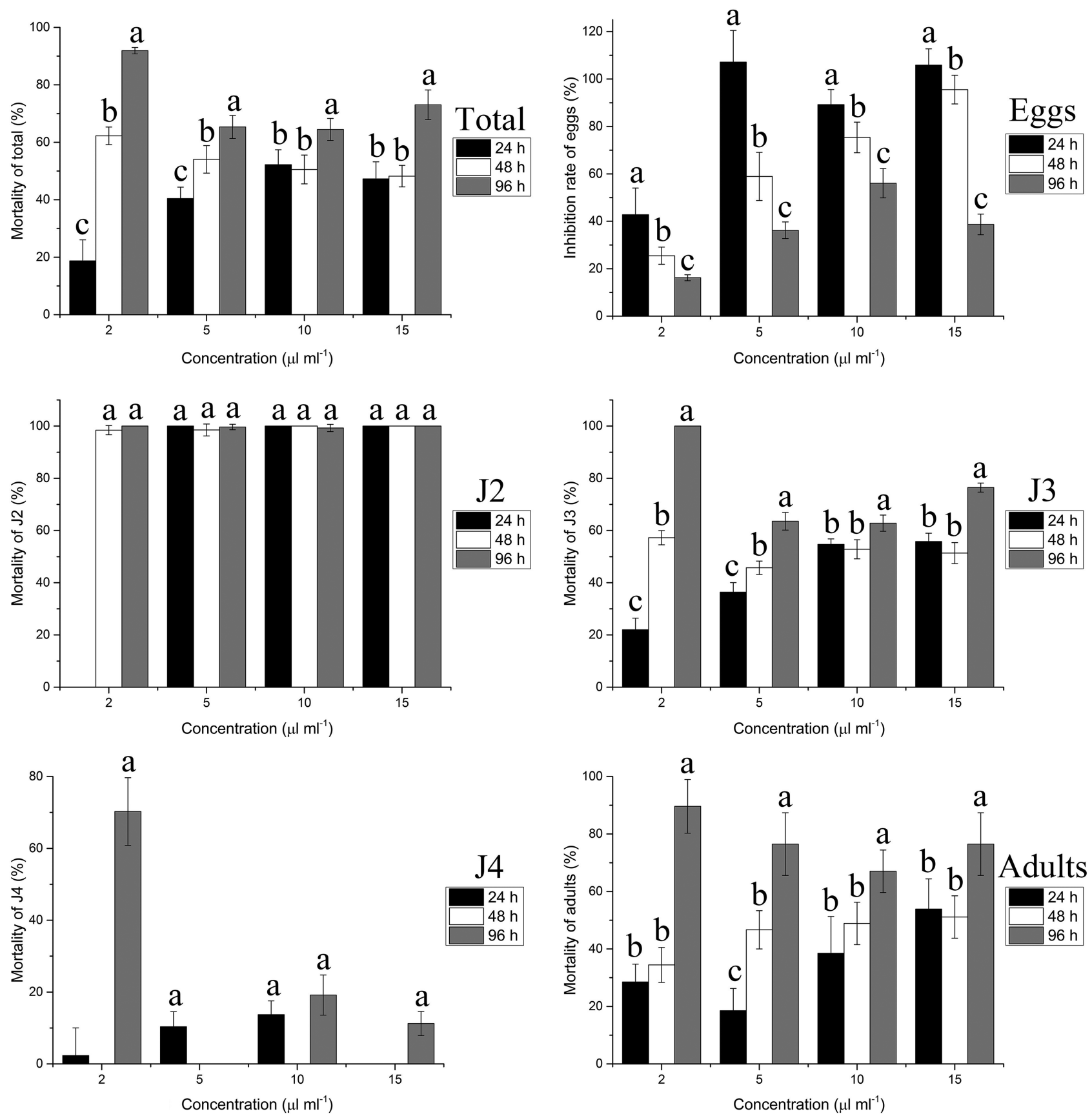

Fig. 5. The mortality and hatch inhibition of different stages of Bursaphelenchus xylophilus after treated with different concentrations $\left(2,5,10\right.$ and $\left.15 \mu \mathrm{g} \mathrm{ml}^{-1}\right)$ of 1,8-cineole at different times $(24,48$ and $96 \mathrm{~h})$ in the dark at $25^{\circ} \mathrm{C}$. Error bars $=\mathrm{SD}$ of the mean. Bars with the same letter are not significantly different based on the independent sample $t$-test. $\mathrm{J} 2=$ second-stage juveniles; J3 $=$ third-stage juveniles; J4 = fourth-stage juveniles.

ta, P. vulnus and Xiphinema index. However, eggs exposed to volatile gases of the Annulohypoxylon sp. were weakly affected, possibly because 1,8-cineole was almost insoluble in water (Amrine et al., 1996), so the slight amount of 1,8-cineole in water did not reach the concentration required to inhibit egg development. The effect of 

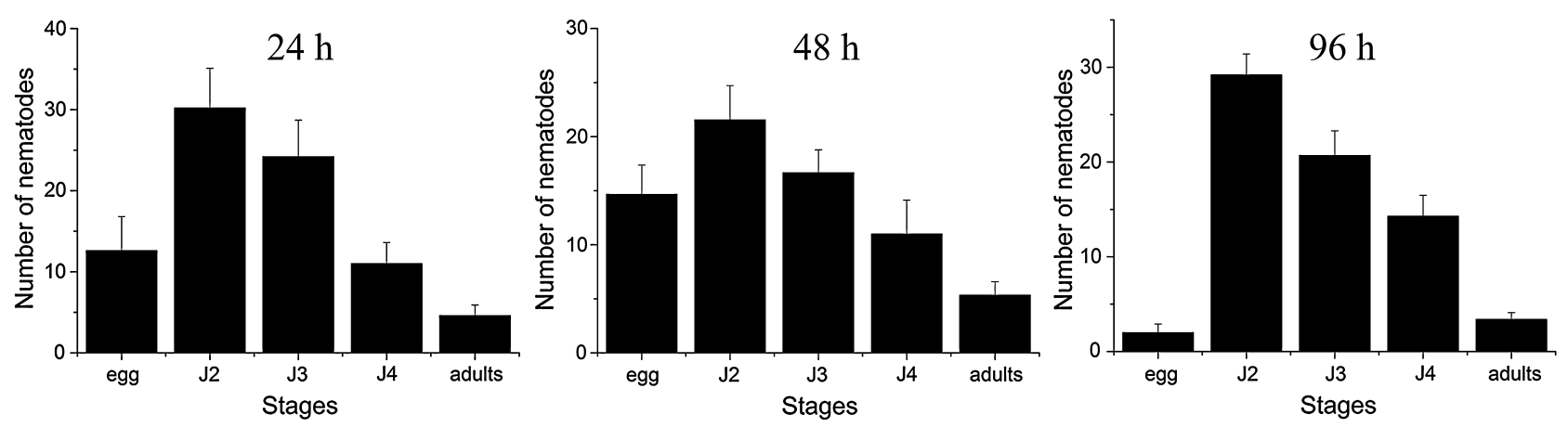

Fig. 6. A $0.5 \%$ Triton X-100 solution was included in the experiments as a control. Numbers of Bursaphelenchus xylophilus in the control treatments after 14,48 and $72 \mathrm{~h}$ in the dark at $25^{\circ} \mathrm{C}$. Nematode populations developed normally. $\mathrm{J} 2=$ second-stage juveniles; $\mathrm{J} 3=$ third-stage juveniles; $\mathrm{J} 4=$ fourth-stage juveniles.

1,8-cineole was not obvious at low concentration but at high concentrations it showed remarkable antifungal effects against all the fungal isolates of chickpea (Shukla $e t$ al., 2012).

In summary, the volatile gas produced by Annulohypoxylon sp. FPYF3050 was able to cause mortality of PWN and 1,8-cineole may be the main active substance. Using nematicidal VOCs of fungi in this way should reduce the environmental damage caused by applying chemical nematicides. This study also contributed to the screening of volatile gases emitted by fungi that can control nematodes. The Annulohypoxylon sp. fungus comes from plants and can grow on a variety of agricultural and forestry residues, which may provide long-term biological control effects after being introduced to the natural environment (Wang et al., 2017). Our results suggested that nematicidal VOCs could be used to develop novel nematicidal agents through further empirical research. However, the nematicidal mechanism of this gas is still unclear, and this needs to be explored in more in-depth future research.

\section{Acknowledgements}

The authors thank Pengfei Wei, Jing Cui and Zhengkai Liu for their assistance in collecting the nematodes (eggs and J2); Wei Zhang in drawing and in vitro experiment; Yuqian Feng in statistical analysis. This project was financially supported by the National Key R\&D Program of China (2017YFD0600100) and Fundamental Research Funds of CAF (CAFYBB2017MA010).

\section{References}

Amrine, J., Noel, B., Mallow, H., Stasny, T. \& Skidmore, R. (1996). Essential oils used to control mites in honey bees. https://www.researchgate.net/publication/228516466 (accessed 19 May 2019).

Andrés, M.F., González-Coloma, A., Sanz, J., Burillo, J. \& Sainz, P. (2012). Nematicidal activity of essential oils: a review. Phytochemistry Reviews 11, 371-390. DOI: 10.1007/ s11101-012-9263-3

Avato, P., Laquale, S., Argentieri, M.P., Lamiri, A., Radicci, V. \& D'Addabbo, T. (2017). Nematicidal activity of essential oils from aromatic plants of Morocco. Journal of Pest Science 90, 711-722. DOI: 10.1007/s10340-016-0805-0

Cheng, H., Lin, M. \& Li, W. (1983). [Pine wilt disease found on the Pinus thunbergii in Nanjing.] Forest Pests and Diseases 4, 1-5.

Cheng, L., Xu, S., Xu, C., Lu, H., Zhang, Z., Zhang, D., Mu, W. \& Liu, F. (2017). Effects of trans-2-hexenal on reproduction, growth and behaviour and efficacy against the pinewood nematode, Bursaphelenchus xylophilus. Pest Management Science 73, 888. DOI: $10.1002 /$ ps.4360

Freire, E.S., Campos, V.P., Pinho, R.S.C., Oliveira, D.F., Faria, M.R., Pohlit, A.M., Noberto, N.P., Rezende, E.L., Pfenning, L.H. \& Silva, J.R.C. (2012). Volatile substances produced by Fusarium oxysporum from coffee rhizosphere and other microbes affect Meloidogyne incognita and Arthrobotrys conoides. Journal of Nematology 44, 321-328.

Giblin-Davis, R.M. (1993). Interactions of nematodes with insects. In: Wajid Khan, M. (Ed.). Nematode interactions. Dordrecht, The Netherlands, Springer, pp. 302-334. DOI: 10. 1007/978-94-011-1488-2_15

Jackson, M.A., Dunlap, C.A. \& Jaronski, S.T. (2009). Ecological considerations in producing and formulating fungal entomopathogens for use in insect biocontrol. BioControl 55, 129-145. DOI: 10.1007/s10526-009-9240-y

Liang, J.Y., Liu, Y., Zhang, X.-X., Zhang, L.-J., Chen, Y., Li, Y., Zhang, H., Kong, W.B. \& Du, S.S. (2018). Antagonistic activ- 
ity of essential oils and their main constituents extracted from Ajania fruticulosa and A. potaninii against Ditylenchus destructor. Nematology 20, 911-916. DOI: 10.1163/1568541100003185

Liang, X., Liu, Z.Y., Kai, Z., Quan, L., Liang, J. \& Zhang, X.Y. (2013). Characterization of the Pinus massoniana transcriptional response to Bursaphelenchus xylophilus infection using suppression subtractive hybridization. International Journal of Molecular Sciences 14, 11356-11375. DOI: 10.3390/ ijms 140611356

Lopez-Llorca, L.V., Maciá-Vicente, J.G. \& Jansson, H.-B. (2007). Mode of action and interactions of nematophagous fungi. In: Ciancio, A. \& Mukerji, M.G. (Eds). Integrated management and biocontrol of vegetable and grain crops nematodes. Dordrecht, The Netherlands, Springer, pp. 51-76. DOI: 10.1007/978-1-4020-6063-2_3

Macías-Rubalcava, M.L., Hernández-Bautista, B.E., Oropeza, F., Duarte, G., González, M.C., Glenn, A.E., Hanlin, R.T. \& Anaya, A.L. (2010). Allelochemical effects of volatile compounds and organic extracts from Muscodor yucatanensis, a tropical endophytic fungus from Bursera simaruba. Journal of Chemical Ecology 36, 1122-1131. DOI: 10.1007/s10886010-9848-5

Morath, S.U., Hung, R. \& Bennett, J.W. (2012). Fungal volatile organic compounds: a review with emphasis on their biotechnological potential. Fungal Biology Reviews 26, 73-83. DOI: 10.1016/j.fbr.2012.07.001

Pimenta, L., Ferreira, M.A., Pedroso, M.P. \& Campos, V.P. (2017). Wood-associated fungi produce volatile organic compounds toxic to root-knot nematode. Scientia Agricola 74, 303-310. DOI: 10.1590/1678-992X-2015-0472

Rossi, Y.E. \& Palacios, S.M. (2015). Insecticidal toxicity of Eucalyptus cinerea essential oil and 1,8-cineole against Musca domestica and possible uses according to the metabolic response of flies. Industrial Crops and Products 63, 133-137. DOI: 10.1016/j.indcrop.2014.10.019

Shaw, J.J., Berbasova, T., Sasaki, T., Jefferson-George, K., Spakowicz, D.J., Dunican, B.F., Portero, C.E., NarváezTrujillo, A. \& Strobel, S.A. (2015). Identification of a fungal 1,8-cineole synthase from Hypoxylon sp. with specificity determinants in common with the plant synthases. Journal of Biological Chemistry 290, 8511-8526. DOI: 10.1074/jbc. M114.636159
Shih, J.Y. \& Tzean, S.S. (1999). Esteya, a new nematophagous genus from Taiwan, attacking the pinewood nematode (Bursaphelenchus xylophilus). Mycological Research 103, 242248. DOI: 10.1017/S0953756298006984

Shinya, R., Takeuchi, Y. \& Futai, K. (2009). A technique for separating the developmental stages of the propagative form of the pine wood nematode, Bursaphelenchus xylophilus. Nematology 11, 305-307. DOI: 10.1163/156854108x399164

Shukla, R., Singh, P., Prakash, B. \& Dubey, N.K. (2012). Antifungal, aflatoxin inhibition and antioxidant activity of Callistemon lanceolatus (Sm.) Sweet essential oil and its major component 1,8-cineole against fungal isolates from chickpea seeds. Food Control 25, 27-33. DOI: 10.1016/j. foodcont.2011.10.010

Strobel, G.A., Dirkse, E. \& Sears, J. (2001). Volatile antimicrobials from Muscodor albus, a novel endophytic fungus. Microbiology 147, 2943-2950. DOI: 10.1099/00221287-14711-2943

Tsao, R. \& Yu, Q. (2000). Nematicidal activity of monoterpenoid compounds against economically important nematodes in agriculture. Journal of Essential Oil Research 12, 350-354. DOI: 10.1080/10412905.2000.9699533

Ulzurrun, V.D.D. \& Hsueh, Y.P. (2018). Predator-prey interactions of nematode-trapping fungi and nematodes: both sides of the coin. Applied Microbiology and Biotechnology 102, 1-11. DOI: $10.1007 / \mathrm{s} 00253-018-8897-5$

Wang, K.Y., Strobel, G.A. \& Yan, D.-H. (2017). The production of 1,8-cineole, a potential biofuel, from an endophytic strain of Annulohypoxylon sp. FPYF3050 when grown on agricultural residues. Journal of Sustainable Bioenergy Systems 7, 65-84. DOI: 10.4236/jsbs.2017.72006

Yan, D.-H., Song, X., Li, H., Luo, T., Dou, G. \& Strobel, G.A. (2018). Antifungal activities of volatile secondary metabolites of four Diaporthe strains isolated from Catharanthus roseus. Journal of Fungi 4, 65. DOI: 10.3390/jof4020065

Yang, Z., Yu, Z., Lei, L., Xia, Z., Shao, L., Zhang, K. \& Li, G. (2012). Nematicidal effect of volatiles produced by Trichoderma sp. Journal of Asia-Pacific Entomology 15, 647650. DOI: 10.1016/j.aspen.2012.08.002

Zhu, N., Bai, L., Schütz, S., Liu, B., Liu, Z., Zhang, X., Yu, H. \& Hu, J. (2016). Observation and quantification of mating behavior in the pinewood nematode, Bursaphelenchus xylophilus. Journal of Visualized Experiments, e54842. DOI: $10.3791 / 54842$ 


\section{Supplementary material}

Table S1. The experiment with mixed-stages (see Materials and methods); the numbers of Bursaphelenchus xylophilus remaining after treated with different concentrations of 1,8-cineole at different times.

\begin{tabular}{|c|c|c|c|c|c|c|c|}
\hline Time (h) & Dose $\left(\mu \mathrm{g} \mathrm{ml}^{-1}\right)$ & Eggs & $\mathrm{J} 2$ & $\mathrm{~J} 3$ & $\mathrm{~J} 4$ & Adults & Total \\
\hline 0 & water & $25.3 \pm 2.8$ & $25 \pm 5.3$ & $32.3 \pm 5.8$ & $10 \pm 2.4$ & $5 \pm 0.8$ & $97.7 \pm 6.1$ \\
\hline \multirow[t]{5}{*}{24} & Triton $\mathrm{X}-100$ & $12.6 \pm 4.2$ & $30.2 \pm 4.9$ & $24.2 \pm 4.5$ & $11 \pm 2.6$ & $4.6 \pm 1.3$ & $82.6 \pm 6.5$ \\
\hline & 2 & $10.8 \pm 3.7$ & $33 \pm 6.1$ & $25.1 \pm 3.1$ & $9.8 \pm 1.7$ & $3.7 \pm 1.2$ & $82.3 \pm 8.6$ \\
\hline & 5 & $26.7 \pm 5.2$ & $0 \pm 0$ & $20.6 \pm 3.1$ & $9 \pm 2.8$ & $4.1 \pm 1.3$ & $60.3 \pm 4.9$ \\
\hline & 10 & $22.1 \pm 5$ & $0 \pm 0$ & $14.6 \pm 3.2$ & $8.7 \pm 1.6$ & $3.1 \pm 1.5$ & $48.4 \pm 6$ \\
\hline & 15 & $26.1 \pm 3.4$ & $0 \pm 0$ & $14.3 \pm 2.5$ & $10.7 \pm 3$ & $2.3 \pm 0.7$ & $53.4 \pm 6.8$ \\
\hline \multirow[t]{5}{*}{48} & Triton X-100 & $14.7 \pm 2.7$ & $21.6 \pm 3.2$ & $16.7 \pm 2.1$ & $11 \pm 3.1$ & $5.3 \pm 1.2$ & $69.2 \pm 4.5$ \\
\hline & 2 & $6.3 \pm 2.6$ & $0.4 \pm 0.5$ & $13.9 \pm 4.8$ & $14.2 \pm 3.3$ & $3.3 \pm 0.9$ & $38.2 \pm 3.6$ \\
\hline & 5 & $14.7 \pm 4.1$ & $0.4 \pm 0.7$ & $17.4 \pm 2.7$ & $11.3 \pm 4$ & $2.7 \pm 1.2$ & $46.6 \pm 5.5$ \\
\hline & 10 & $20.7 \pm 5.3$ & $0 \pm 0$ & $15.2 \pm 4.1$ & $12.9 \pm 3$ & $2.6 \pm 0.8$ & $51.3 \pm 6.7$ \\
\hline & 15 & $23.6 \pm 3.1$ & $0 \pm 0$ & $15.6 \pm 3.6$ & $10.9 \pm 3.6$ & $2.4 \pm 0.8$ & $52.4 \pm 4.5$ \\
\hline \multirow[t]{5}{*}{96} & Triton X-100 & $2 \pm 0.9$ & $29.2 \pm 2.2$ & $20.7 \pm 2.6$ & $14.3 \pm 2.2$ & $3.4 \pm 0.7$ & $69.7 \pm 6.3$ \\
\hline & 2 & $4 \pm 1.5$ & $0 \pm 0$ & $0 \pm 0$ & $3 \pm 1.6$ & $0.6 \pm 0.5$ & $8.2 \pm 5.2$ \\
\hline & 5 & $9 \pm 2.8$ & $0.1 \pm 0.3$ & $11.9 \pm 2.6$ & $12.9 \pm 3.2$ & $1.2 \pm 1$ & $35.1 \pm 4.5$ \\
\hline & 10 & $17.6 \pm 5.2$ & $0.2 \pm 0.4$ & $12.1 \pm 3.4$ & $8.1 \pm 2.7$ & $1.7 \pm 0.8$ & $39.7 \pm 3.2$ \\
\hline & 15 & $9.6 \pm 2.8$ & $0 \pm 0$ & $7.7 \pm 2.9$ & $8.9 \pm 1.3$ & $1.2 \pm 0.6$ & $27.3 \pm 5.6$ \\
\hline
\end{tabular}

Values are means $\pm \mathrm{SD}$ of the mean. $\mathrm{J} 2=$ second-stage juveniles; $\mathrm{J} 3=$ third-stage juveniles; J4 = fourth-stage juveniles. 